\title{
On the non-linearity of the subsidiary systems
}

\author{
Helmut Friedrich \\ Albert-Einstein-Institut \\ Max-Planck-Institut für Gravitationsphysik \\ Am Mühlenberg 1 \\ 14476 Golm \\ Germany
}

November 8, 2018

\begin{abstract}
In hyperbolic reductions of the Einstein equations the evolution of gauge conditions or constraint quantities is controlled by subsidiary systems. We point out a class of non-linearities in these systems which may have the potential of generating catastrophic growth of gauge resp. constraint violations in numerical calculations.
\end{abstract}

\section{Introduction}

Most numerical calculations of solutions to Einstein's field equations are being plagued by an undesirably fast growth of constraint violations. A huge variety of reduced equations has been derived from Einstein's equations with the hope of finding versions with stable propagation properties and there have been suggested modifcations of the equations which were hoped to force back the solution to the constraint manifold (we refer to [6] for further dicussion and references). More recently, there have been performed stability analyses of the subsidiary systems which control the evolution of the gauge conditions or the constraint quantities (cf. [1], 8] and the references given there). These led to requirements on the coefficients of the equations which in the case of 1 resulted in geometric conditions on the foliation underlying the evolution by the main system. Nevertheless, the field appears to be wide open, most of the suggested remedies are 
experimental, and the cause of the problems is not understood. In [1], 8] the subsidiary systems have been considered as linear systems on given space-times. In contrast, we wish to emphasize in this note the non-linearity of the subsidiary system, which appears to have a potential of generating catastrophic constraint violations. To understand the extent to which these non-linearities may affect the numerical construction of space-times and to develop, if necessary, ways to avoid their effects, further investigations are needed.

\section{The hyperbolic reduction}

We shall use a few facts about the hyperbolic reduction procedure by which the geometric initial value problem for Einstein's field equations is reduced to a Cauchy problem for a hyperbolic system. There exist now many versions of it, whose general underlying structure are, however, more or less the same. Because it leads to concise expressions, we will use the metric coeffficients as basic unknowns and consider the representation of the field equations in which their evolution is governed by a system of wave equations. To emphasize the independence of our discussion of any particular coordinate system we shall employ the notion of a gauge source function introduced in [2]. We refer to [3], 4 for more details on the reduction procedure.

Let $(M, g)$ denote a smooth 4-dimensional Lorentz space with smooth spacelike Cauchy hypersurface $S$ and $U \ni x^{\lambda} \rightarrow F^{\mu}\left(x^{\lambda}\right) \in V$ a smooth map of an open subset $U$ into another subset $V$ of $\mathbb{R}^{4}$. With functions $x^{\mu}$ and their (4dimensional) differentials $d x^{\nu}$ prescribed on some open subset $W$ of $S$ one can solve near $W$ the Cauchy problem for the semi-linear system of wave equations

$$
\nabla_{\nu} \nabla^{\nu} x^{\mu}=-F^{\mu}\left(x^{\lambda}\right)
$$

If the $d x^{\mu}$ have been chosen linearly independent on $W$ the solution will provide a smooth coordinate system $x^{\lambda}$ on some neighbourhood of $W$ in $M$. In terms of these coordinates the equations above take the form

$$
-\Gamma^{\mu}\left(x^{\lambda}\right)=-F^{\mu}\left(x^{\lambda}\right)
$$

where the $\Gamma^{\mu}$ denote the contracted Christoffel symbols $\Gamma^{\mu}=g^{\nu \eta} \Gamma_{\nu}{ }^{\mu}{ }_{\eta}$ of $g_{\mu \nu}$. This shows (ignoring subtleties arising in situations of low differentiability) that the contracted Christoffel symbols can locally be made to agree with any prescribed set of functions $F^{\mu}$ and that these function and the initial data determine the coordinates uniquely. We refer to these functions as gauge source functions. Assume now that $(M, g)$ is to be obtained by solving a Cauchy problem for Einstein's vacuum field equations. We shall derive the properties which will help us formulate this problem as a Cauchy problem for hyperbolic equations. The contracted Christoffel symbols are of particular interest to us because the Ricci tensor of $g$ can be written in the form

$$
R_{\mu \nu}=-\frac{1}{2} g^{\lambda \rho} g_{\mu \nu, \lambda \rho}+\nabla_{(\mu} \Gamma_{\nu)}+\Gamma_{\lambda}{ }_{\mu} g_{\eta \delta} g^{\lambda \rho} \Gamma_{\rho}{ }_{\nu}{ }_{\nu}+2 \Gamma_{\delta}{ }^{\lambda}{ }_{\eta} g^{\delta \rho} g_{\lambda(\mu} \Gamma_{\nu)}{ }_{\rho}{ }_{\rho}
$$


Here the contracted Christoffel symbols (and the functions $F_{\nu}=g_{\nu \mu} F^{\mu}$ considered in the following) are being formally treated as if they defined a vector field (which, of course, they do not). Thus $\Gamma_{\nu}=g_{\nu \mu} \Gamma^{\mu}$ and $\nabla_{\mu} \Gamma_{\nu}=\partial_{\mu} \Gamma_{\nu}-\Gamma_{\mu}{ }_{\nu}{ }_{\nu} \Gamma_{\lambda}$. The discussion above suggests replacing in (2.1) the functions $\Gamma_{\nu}$ by freely chosen gauge source functions $F_{\nu}$ (so that the resulting expression will depend in general on the coordinates $x^{\lambda}$ not any longer only through the $\left.g_{\mu \nu}\right)$. With this replacement the vacuum field equations take the form

$0=R_{\mu \nu}^{F} \equiv-\frac{1}{2} g^{\lambda \rho} g_{\mu \nu, \lambda \rho}+\nabla_{(\mu} F_{\nu)}+\Gamma_{\lambda}{ }_{\mu}{ }_{\mu} g_{\eta \delta} g^{\lambda \rho} \Gamma_{\rho}{ }^{\delta}{ }_{\nu}+2 \Gamma_{\delta}{ }^{\lambda}{ }_{\eta} g^{\delta \rho} g_{\lambda(\mu} \Gamma_{\nu)}{ }_{\rho}{ }_{\rho}$

of a system of wave equations for the $g_{\mu \nu}$. We refer to (2.2) as the main evolution system or the reduced equations. For this system the Cauchy problem for $g_{\mu \nu}$ with data satisfying the constraints on a space-like hypersurface $S$ is well posed. Suppose that $g_{\mu \nu}$ is a solution of this problem near $S$. Since equation (2.2) is in fact of the form

$$
R_{\mu \nu}=\nabla_{(\mu} Q_{\nu)}
$$

where $Q_{\mu}=\Gamma_{\mu}-F_{\mu}$ with the $\Gamma_{\mu}$ calculated from $g_{\mu \nu}$, it is not clear a priori whether the solution $g$ will indeed satisfy the gauge condition $\Gamma_{\mu}=F_{\mu}$ and thus the vacuum field equation $R_{\mu \nu}=0$.

The Bianchi identity, which holds for any metric, implies

$$
0=2 \nabla^{\mu}\left(R_{\mu \nu}-\frac{1}{2} R g_{\mu \nu}\right)=\nabla_{\mu} \nabla^{\mu} Q_{\nu}+R_{\nu}^{\mu} Q_{\mu}
$$

and thus a system of wave equations for the quantities $Q_{\nu}$. We refer to this system as subsidiary system.

The detailed analysis shows that if the Cauchy problem for the main evolution system is arranged so that the initial data satisfy the constraints and the gauge condition $Q_{\nu}=0$ on $S$, it follows for the solution of the main evolution system that also $d Q_{\nu}$ and thus any 'time derivative' $\partial_{t} Q_{\nu}$ transverse to $S$ vanishes on $S$. The uniqueness property of the subsidiary system therefore implies that the solution to the main evolution system does indeed satisfy $Q_{\nu}=0$ on the domain of dependence of $S$ with respect to $g$. This reduces the local Cauchy problem for Einstein's field equations to the problem of solving equation (2.2), which thus takes the central role in the analytic discussion. Of the subsidiary system only the homogeneity and the resulting uniqueness property are needed.

\section{The non-linearity of the subsidiary equation}

The main evolution system is also central in numerical discussions. Because the initial data $Q_{\mu}$ and $\partial_{t} Q_{\nu}$ on the initial hypersurface $S$ come with an error, the evolution properties of the subsidiary system will, however, also become important.

There is no way to relate a numerical solution to the solution of the continuum problem one wants to approximate. To get some idea how errors infiltrate into 
the various systems, it is useful to consider an analogy accessible to analytic methods. We assume the main evolution system to be satisfied by fields $g_{\mu \nu}$ of class $C^{3}$ with an error term $E_{\mu \nu}$ of class $C^{1}$ so that

$$
R_{\mu \nu}=\nabla_{(\mu} Q_{\nu)}+E_{\mu \nu}
$$

Nothing will be assumed about the origin and structure of this error and the errors in the initial data $Q_{\mu}$ and $\partial_{t} Q_{\nu}$ on $S$.

Using the Bianchi identity with (3.1) gives the analogue

$$
\nabla_{\mu} \nabla^{\mu} Q_{\nu}+R_{\nu}^{\mu} Q_{\mu}=-2 \nabla^{\mu}\left(E_{\mu \nu}-\frac{1}{2} g_{\mu \nu} E_{\rho}{ }^{\rho}\right)
$$

of the subsidiary equation (2.4). This equation will in general not be homogeneous any longer. There may be a way to avoid this problem. Assume a splitting of the form

$$
E_{\mu \nu}=\nabla_{(\mu} e_{\nu)}+f_{\mu \nu}
$$

with some $C^{2}$ vector field $e_{\mu}$ and some symmetric $C^{1}$ tensor field $f_{\mu \nu}$. With (3.1) this gives

$$
R_{\mu \nu}=\nabla_{(\mu} Q_{\nu)}^{\prime}+f_{\mu \nu} \quad \text { with } \quad Q_{\nu}^{\prime}=Q_{\nu}+e_{\nu}
$$

If $f_{\mu \nu}$ would vanish this would just amount to a redefinition

$$
F_{\mu} \rightarrow F_{\mu}^{\prime}=F_{\mu}-e_{\mu},
$$

of the gauge source function which may be quite harmless. Moreover, we would get a homogenous wave equation for $Q_{\mu}^{\prime}$. If $E_{\mu \nu}$ is known but not of the form $\nabla_{(\mu} e_{\nu)}$, a homogenous system can be obtained by choosing the splitting suitably. If we require $e_{\mu}$ to solve the system of wave equations

$$
\nabla_{\mu} \nabla^{\mu} e_{\nu}+R_{\nu}^{\mu} e_{\mu}=2 \nabla^{\mu}\left(E_{\mu \nu}-\frac{1}{2} g_{\mu \nu} E_{\rho}^{\rho}\right)
$$

it follows from the splitting above that

$$
\nabla^{\mu}\left(f_{\mu \nu}-\frac{1}{2} g_{\mu \nu} f_{\rho} \rho\right)=0
$$

and the Bianchi identity implies for $Q_{\mu}^{\prime}$ the homogeneous equation

$$
\nabla_{\mu} \nabla^{\mu} Q_{\nu}^{\prime}+R_{\nu}^{\mu} Q_{\mu}^{\prime}=0
$$

One could now try to analyse how errors in $Q_{\mu}$ and $\partial_{t} Q_{\nu}$ on $S$ are propagated, in dependence of $E_{\mu \nu}$, by (3.2) or how errors in $Q_{\mu}^{\prime}$ and $\partial_{t} Q_{\nu}^{\prime}$ on $S$ will be propagated by (3.4). Assuming in this analysis $E_{\mu \nu}$ and $g_{\mu \nu}$ whence $R_{\mu \nu}[g]$ as suitably bounded but given with no further information, standard energy estimates admit but cannot exclude exponential growth of $Q_{\mu}$ resp. $Q_{\mu}^{\prime}$. One might be able to work out conditions on $R_{\mu \nu}[g]$ which will allow one to keep the 
errors under control. However, by itself this will be of limited use. Inserting (3.1) into (3.2) and, observing the splitting, into (3.4), gives

$$
\nabla_{\mu} \nabla^{\mu} Q_{\nu}+Q^{\lambda}\left(\nabla_{(\lambda} Q_{\nu)}+E_{\lambda \nu}\right)=-2 \nabla^{\mu}\left(E_{\mu \nu}-\frac{1}{2} g_{\mu \nu} E_{\rho}{ }^{\rho}\right),
$$

and

$$
\nabla_{\mu} \nabla^{\mu} Q_{\nu}^{\prime}+Q^{\prime \lambda}\left(\nabla_{(\lambda} Q_{\nu)}^{\prime}+f_{\lambda \nu}\right)=0,
$$

respectively. The relative size of the errors $E_{\lambda \nu}$ and $f_{\lambda \nu}$ is not clear. More important is that in this form the subsidiary system shows its non-linearity. If $Q_{\mu}^{\prime}$ and $\partial_{t} Q_{\nu}^{\prime}$ vanish on $S$, equation (3.6) will still imply that $Q_{\mu}^{\prime}$ vanishes in the domain of dependence. If $Q_{\mu}^{\prime}$ and $\partial_{t} Q_{\nu}^{\prime}$ do not vanish on $S$, however, the following observations indicate that (3.6) may imply a growth of the solution $Q_{\mu}^{\prime}$ which is worth than exponential. We assume $g=d t^{2}-\delta_{a b} d x^{a} d x^{b}$ (so that (3.6) decouples from (3.3) $), f_{\lambda \nu}=0$, and initial data which satisfy for $a=1,2,3$,

$$
Q_{a}^{\prime}=0, \quad \partial_{t} Q_{a}^{\prime}=0, \quad \partial_{a} Q_{0}^{\prime}=0, \quad \partial_{a} \partial_{t} Q_{0}^{\prime}=0 \quad \text { on } \quad\{t=0\},
$$

so that the error resides only in the constant functions $a=Q_{0}^{\prime}, b=\partial_{t} Q_{0}^{\prime}$ on $\{t=0\}$.

Equation (3.6) then implies $Q_{a}^{\prime} \equiv 0$ and reduces in fact to $\partial_{t} Q_{0}^{\prime}=\frac{1}{2}(c-$ $\left.Q_{0}^{\prime 2}\right)$ with $c=2 b+a^{2}$. The integration gives $Q_{0}^{\prime}=a=$ const. if $b=0$, $Q_{0}^{\prime}=\sqrt{c} \frac{a+\sqrt{c} \tanh \frac{\sqrt{c} t}{2}}{\sqrt{c}+a \tanh \frac{\sqrt{c} t}{2}}$ if $0 \neq 2 b>-a^{2}, Q_{0}^{\prime}=\frac{2 a}{a t+2}$ if $a^{2}=-2 b$, and $Q_{0}^{\prime}=\sqrt{|c|} \tan \left\{-\frac{\sqrt{|c|} t}{2}+\arctan \frac{a}{\sqrt{|c|}}\right\}$ if $2 b<-a^{2}$. The solutions thus remain bounded for $t \geq 0$ if $b \geq 0$ or if $a \geq 0$ and $-a^{2}<2 b<0$, while they develop poles at some $t_{*}>0$ if $b<0$ and $a \leq 0$ or if $2 b<-a^{2}$ and $a>0$. (If the corresponding initial data would be modified outside the intersection of the hypersurface $\{t=0\}$ with the backward light cone of the point $\left(t_{*}, x^{a}\right)$, the solution would still become singular at $\left(t_{*}, x^{a}\right)$.)

We note that in general any Killing vector field $K$ of a vacuum solution $g_{\mu \nu}$ satisfies equation (2.4) with $Q^{\mu}=K^{\mu}$ in the coordinates $x^{\mu}$. In the present case this gives solutions which grow linearly.

For us the following observation is important:

With our assumption on $g_{\mu \nu}$ it follows that each neighbourhood of the initial data $Q_{a}=0$ and $\partial_{t} Q_{a}=0$ contains initial data for 2.4) for which the solution $Q_{a}\left(x^{\mu}\right)$ become unbounded at some finite $x^{0}=t_{*}>0$. 


\section{Concluding remarks}

The only purpose of the discussion above was to indicate the behaviour of solutions to the subsidiary system. We expect to find a similar singular behaviour of the solutions to equation (2.4) if the latter is given with a general smooth metric $g_{\mu \nu}$ (extending sufficiently far into the future). It would be interesting to know whether the singular data set, i.e. the subset of data which determine singular solutions of equation (2.4), is open or of lower dimension in the set of all data if the metric $g$ is given. While the singular data set itself will depend on the metric $g$, such a characterization may be independent of the chosen metric and may help avoid entering the singular sector.

In a consistent discussion of the growth of $Q_{\mu}$ one would have to consider the main system and the subsidiary system

$$
R_{\mu \nu}=\nabla_{(\mu} Q_{\nu)}, \quad \nabla_{\mu} \nabla^{\mu} Q_{\nu}+Q^{\lambda} \nabla_{(\lambda} Q_{\nu)}=0,
$$

as a coupled system. The main equation can be studied independently. The subsidiary system, implicit in the main system, depends on the metric defined by the latter. If $Q_{\mu}$ tends to grow, the main system will react to it, whether for the better or the worth is not clear. Whether with changing $g_{\mu \nu}$ the nonlinearity of the subsidiary system can still imply a blow up of $Q_{\mu}$ at a finite time needs to be analysed.

For this purpose it might be interesting to study under simplifying assumptions such as spherical symmetry whether the model system

$$
R_{\mu \nu}=\nabla_{(\mu} q_{\nu)}, \quad \nabla_{\mu} \nabla^{\mu} q_{\nu}+q^{\lambda} \nabla_{(\lambda} q_{\nu)}=0,
$$

considered as Einstein equations coupled to a source field given by a vector field $q_{\mu}$, will develop a blow up for suitable data. The reduction of these equations is obtained by a slight modification of the one described above. The system can be simplified further by assuming $q_{\mu}$ to be a differential $q_{\mu}=\nabla_{\mu} f$ of some function $f$. The second equation will then be implied if $f$ satisfies

$$
\nabla_{\mu} \nabla^{\mu} f+\nabla_{\mu} f \nabla^{\mu} f=\text { const. }
$$

which is a wave map equation in the case where the constant on the right hand side vanishes. In any case the results might lead to an identifcation of a mechanism responsible for the growth of constraint violations and to the development of methods to avoid them.

Some authors add terms built from $Q_{\nu}$ to the main system, which appear to reduce the growth of the constraint violations in certain calculation [5], [7]. This may be related to the different effects of the non-linearities which are obtained in the appropriately modified subsidiary systems.

There are available now many different types of reductions. Depending on several choices, the subsidiary system may control the preservation of the gauge or the preservation of constraints or a mixture thereof. In spite of the different appearance of the resulting main and subsidiary systems we expect that similar 
non-linearities as the one discussed above will occur in any subsidiary system, though, depending on the system, they may have different effects.

In a numerical scheme for the second order wave equations the subsidiary system, which is of third order in the metric, can, of course, hardly be identified any longer as a kind of identity and the relations between the two systems is obscured. But if the non-linearity of the subsidiary system can have for nonvanishing initial data $Q_{\mu}$ and $\partial_{t} Q_{\mu}$ drastic effects in the continuum model, they are likely to be reflected in numerical calculations.

\section{References}

[1] J. Frauendiener, T. Vogel. On the stability of constraint propagation. http://de.arXiv.org/abs/gr-qc/0410100

[2] H. Friedrich. On the hyperbolicity of Einstein's and other gauge field equations. Comm. Math. Phys. 100 (1985) 525-543.

[3] H. Friedrich. Hyperbolic Reductions for Einstein's Equations. Class. Quantum Grav. 13 (1996) 1451-1469.

[4] H. Friedrich, A. Rendall. The Cauchy Problem for the Einstein Equations. In: B. Schmidt (ed.): Einstein's Field Equations and Their Physical Implications. Springer, Lecture Notes in Physics, Berlin 2000.

[5] C. Gundlach. Private communication.

[6] L. Lehner, O. Reula. Status quo and open problems in the numerical construction of space-times. In: P. T. Chruściel, H. Friedrich (eds.): The Einstein equations and the large scale behaviour of gravitational fields. Birkhäuser, Basel, 2004.

[7] F. Pretorius. Private communication.

[8] G. Yoneda, H. Shinkai. Diagonalizability of constraint propagation matrices. Class. Quantum Grav. 20 (2003) L31 - L36. 